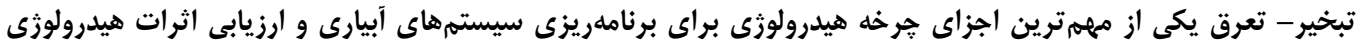

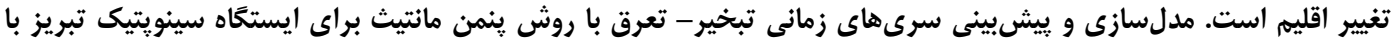

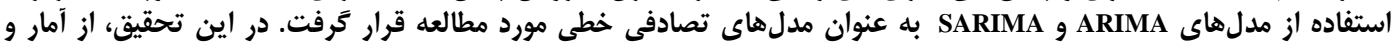

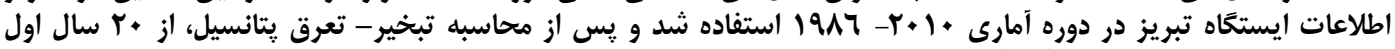

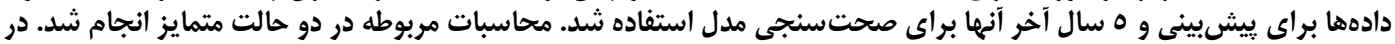

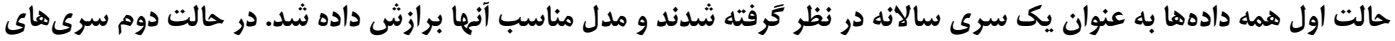

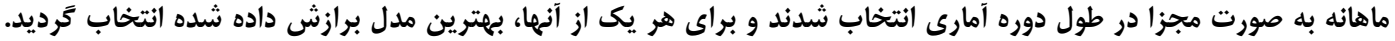

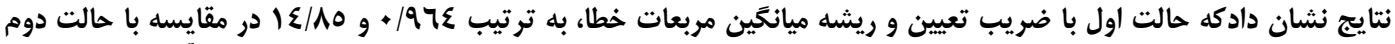

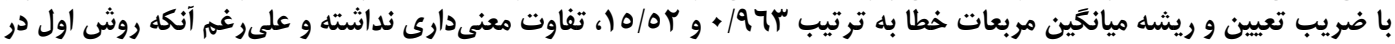

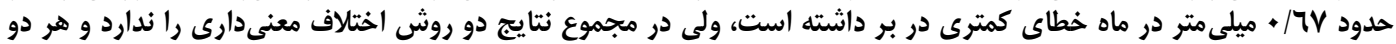

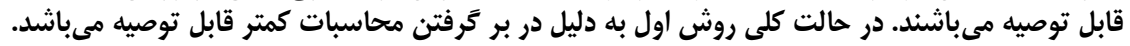

وازههاى كليدى: يِيشبينى، ينمن مانتيث، ARIMA و SARIMA

مدلهاى ساده تجربى تا روشهاى يِيجِيده تركيبى (مانند

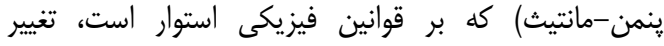

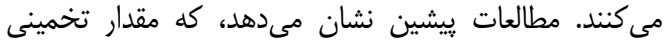

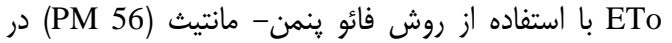

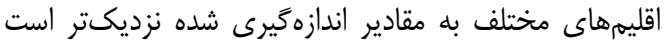

به علت يِيجيدگى و عدم دانش كافى در مورد فر آيندهاى فيزيكى در خرخه هيدرولوزيك از جمله تبخير - تعرق، ساخت إند

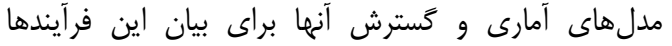

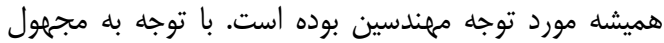

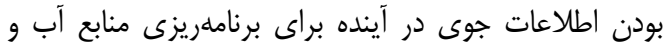

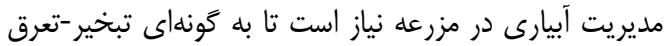

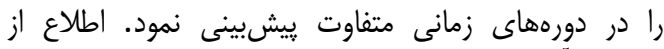

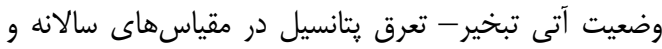

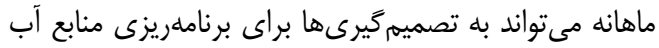

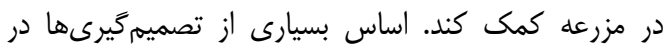

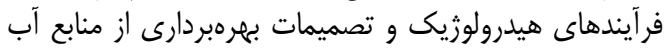

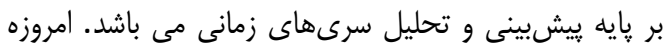

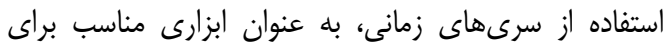

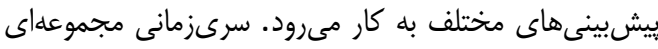

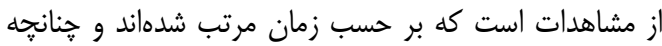

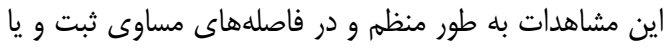

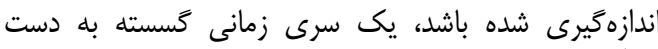

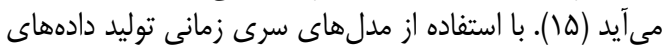

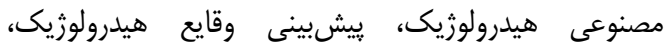

تبخير- تعرق ' يكى از مؤلفههاى مهمم خرخه هيدرولوزى

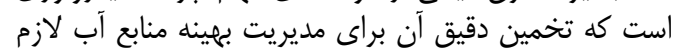

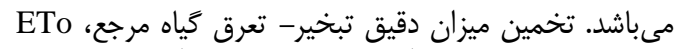

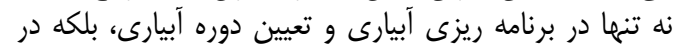

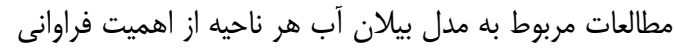

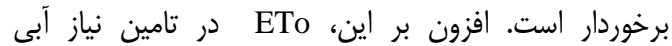

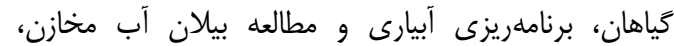

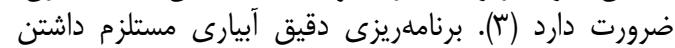

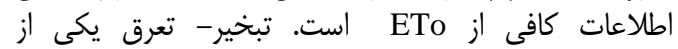

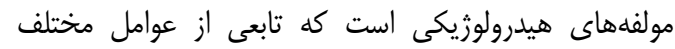

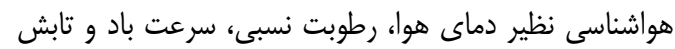

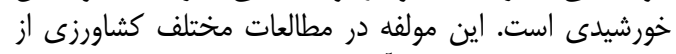

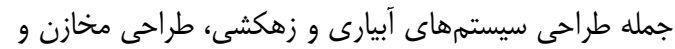

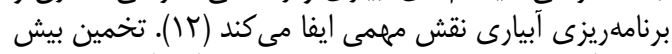

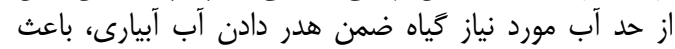

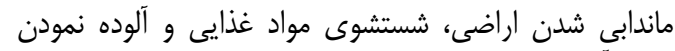

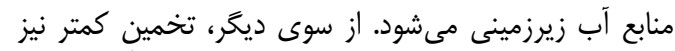

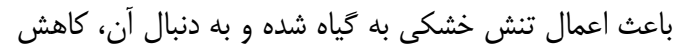

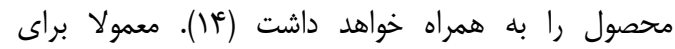

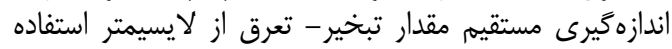

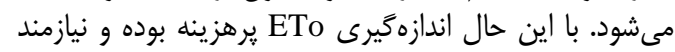

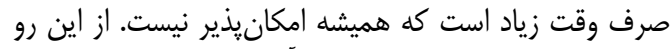

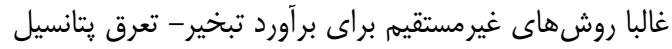

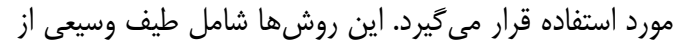


آتى با استفاده از الكوى آريماى فصلى بيشبيشى بينى كردند.

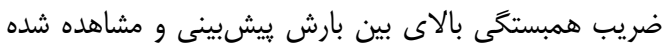

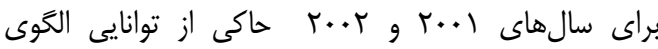

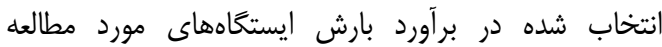

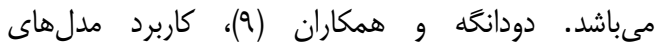

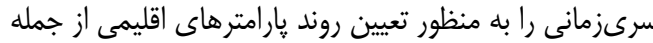

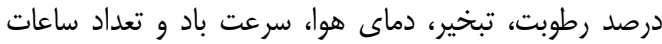

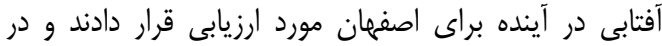

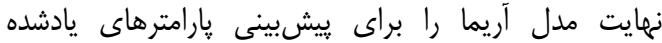

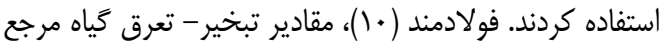

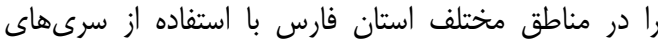

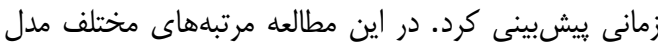

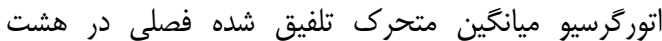

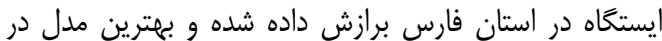

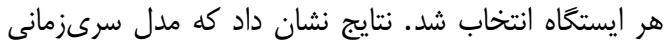

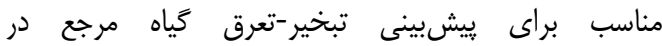
ايستخاههاى مختلف يكسان نمى باشد. شيروانى و هنر تينر (19)،

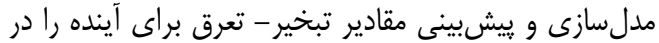

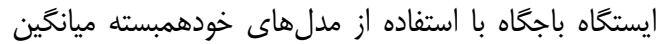

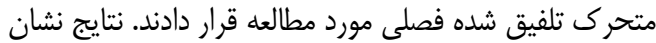

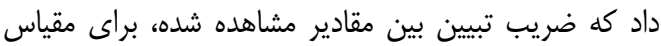

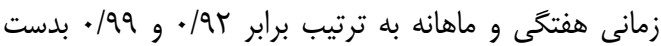

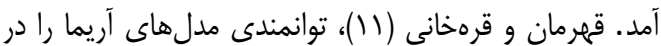

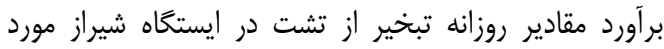

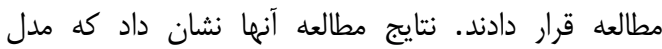

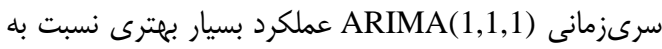

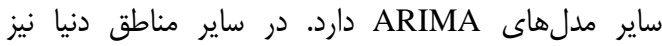

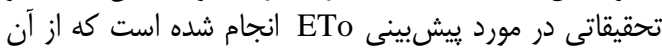

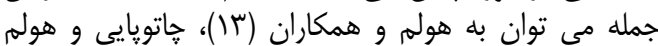

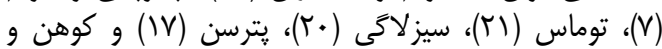

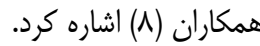

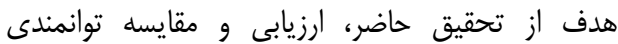

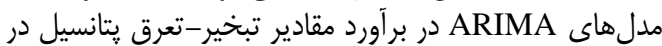

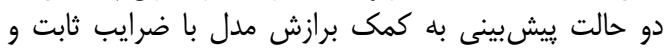
ييشبينى به كمك برازش مدل با ضرايب متغير مىباشد.

\section{مواد و روشها}

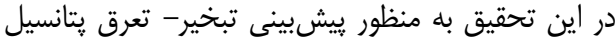

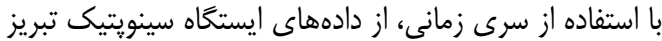

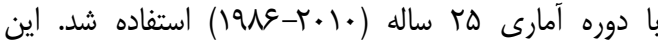

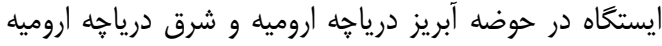

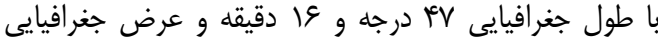

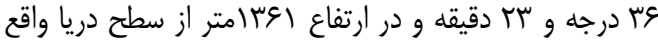

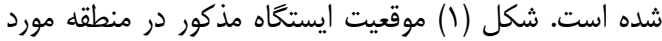

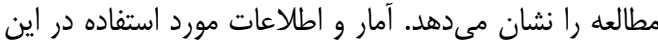

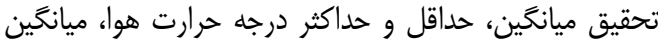

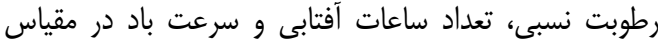

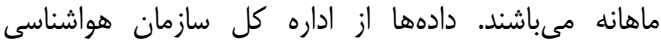

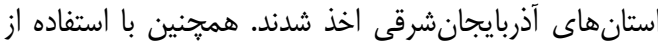

تشخيص روند و يرش در دادهها، تكميل خلا آمارى و تطويل

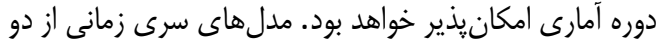

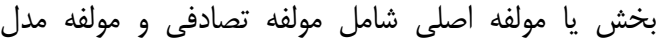

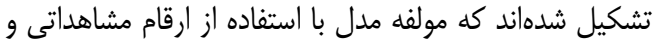

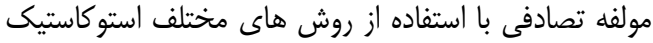

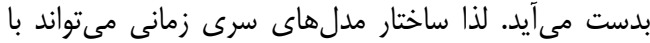

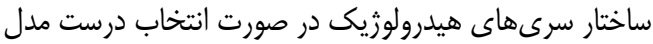

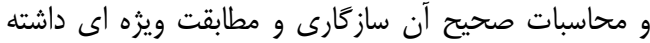

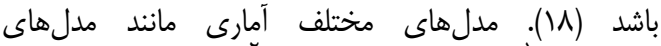

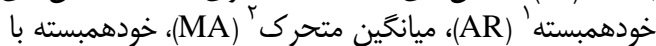

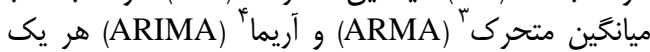

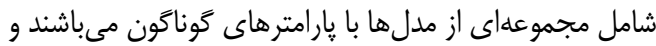

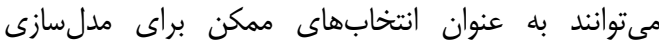

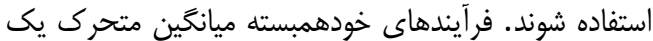

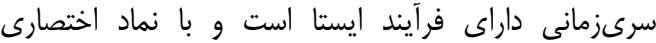
ARMA(p,q) خودهمبسته و q مرتبه ميانخين متحرك است. از طرف دان ديخر

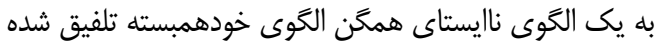

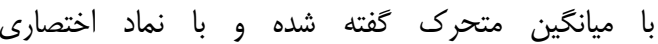
ARIMA(p,d,q) تفاضلى است كه براى تبديل سرى ناليستا به سرى سرى ايستا

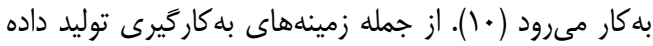

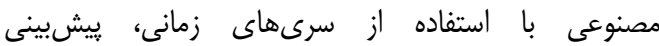
تبخير- تعرق يتانسيل مىباشد.

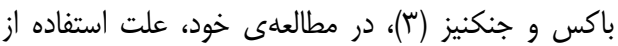

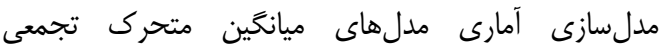

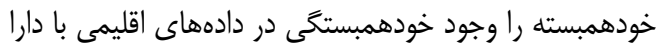

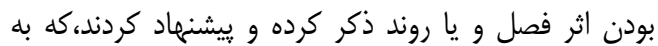

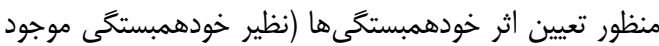

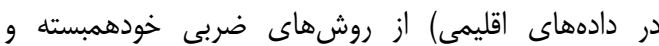

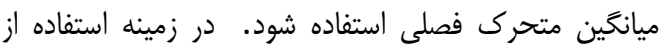

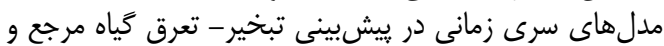

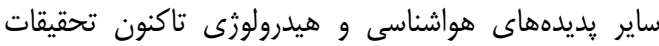

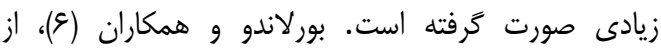

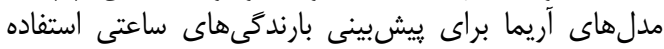

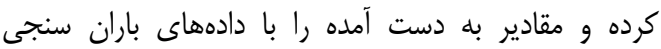

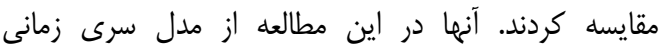
ARIMA $(1,1,0)$

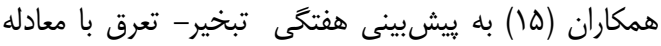

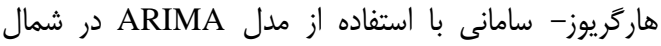

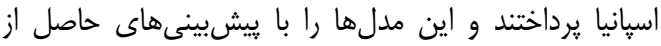

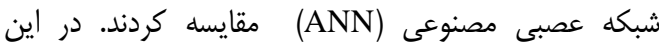

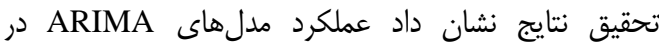

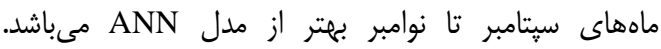

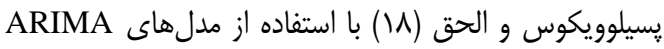

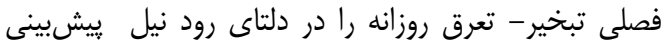

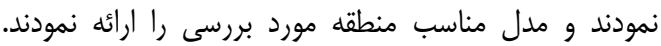

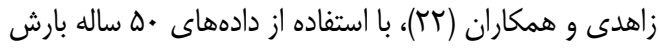

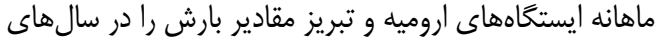


در اين رابطه: - مابخ:

تابش

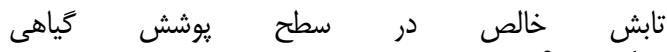
(MJ.m $\left.m^{-2} d a y^{-1}\right)$ r مترى إز

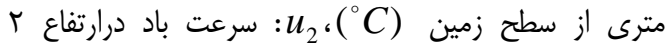

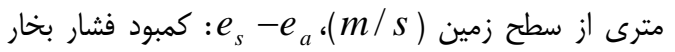

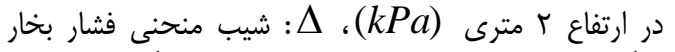

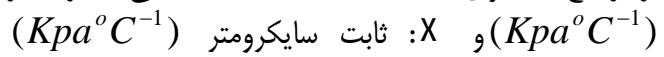
مى باشد.
آزمون ران- تست از همكَى دادهها اطمينان به عمل آمد.

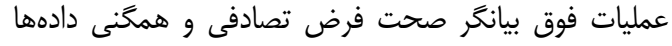

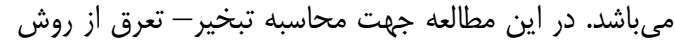

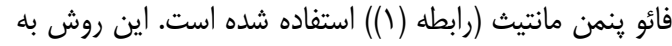

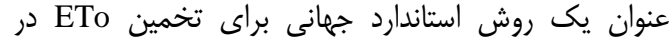

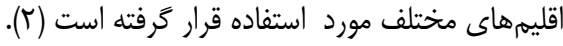

ETo $=\frac{0.408 \Delta\left(R_{n}-G\right)+\gamma \frac{900}{T+273} u_{2}\left(e_{s}-e_{a}\right)}{\Delta+\gamma\left(1+0.34 u_{2}\right)}$

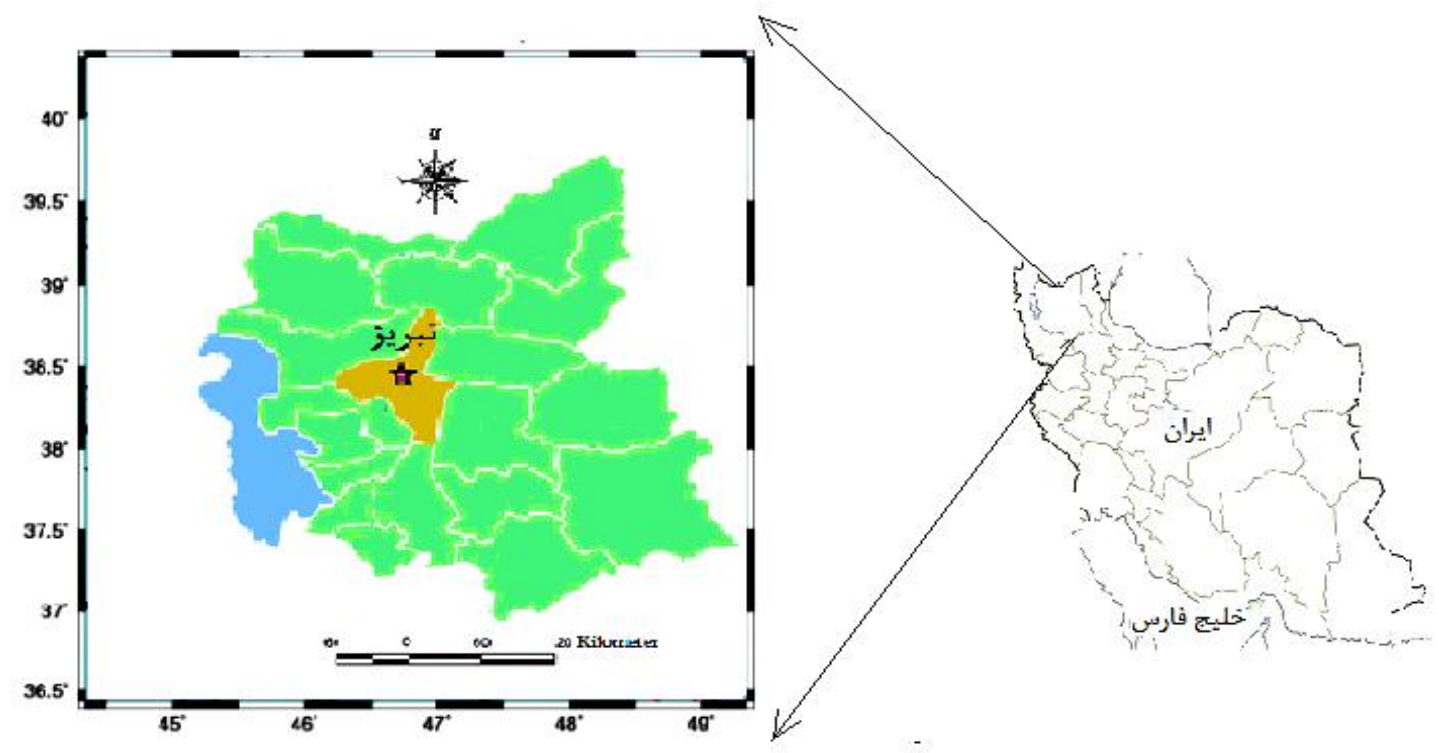

شكل 1- موقعيت منطقه مورد مطالعه در استان آذربايجان شرقى و ايران

Figure 1. The geographical location of the study area in the East Azarbaijan, Iran

اكر هر داده ثبت شده سرىزمانى t با زمان قبل و ويا زمان بعد إندان

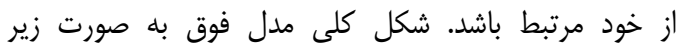

$Z_{t}=\sum_{i=1}^{P}\left(\varphi_{i} Z_{t-i}\right)+\varepsilon_{t}$

مىباشد.

در اين رابطه p رسته يا مرتبه مدل و و وان ها ضرايب مدل

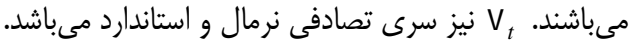

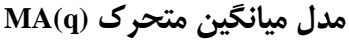

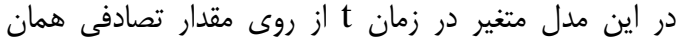

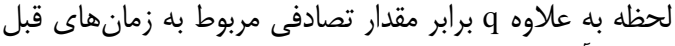

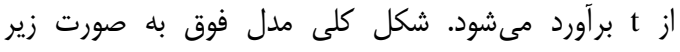

$Z_{t}=\sum_{i=1}^{q}\left(\theta_{j} \varepsilon_{t-j}\right)+\varepsilon_{t}$

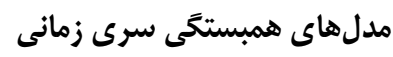

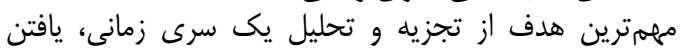

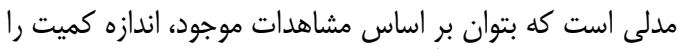

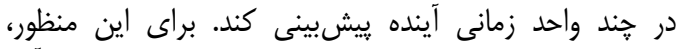

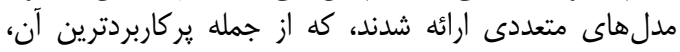

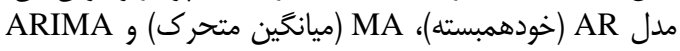

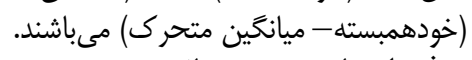

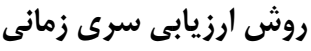

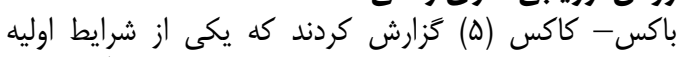

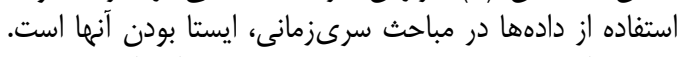

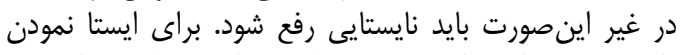

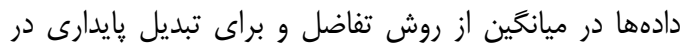
واريانس از روش باكس - كاكس استفاده مى شئود.

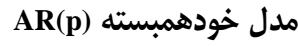

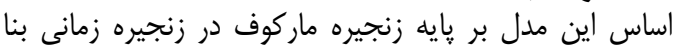

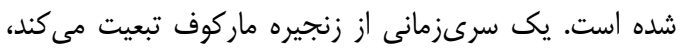


14. برآورد تبخير - تعرق يّانسيل بر اساس مدلهاى تصادفى سرىمانى

جهت انتخاب بهترين مدل در مطالعه حاضر از معيار آكائيك

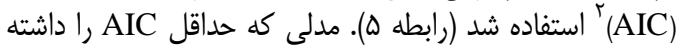

$$
A I C=-2 L N(L)+2 m
$$

كه، ( $)$

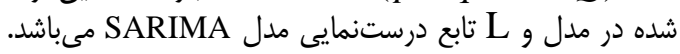

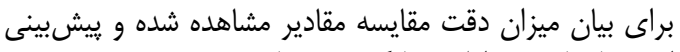

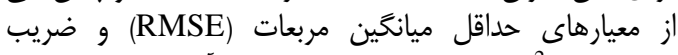
همبستخى (R2) استفاده شد. شكل كلى آنها به صورت زير

$$
\begin{aligned}
& R M S E=\sqrt{\frac{\sum\left(X_{0}-X_{p}\right)^{2}}{n}} \\
& R^{2}=\frac{\sum\left(X_{0}-\overline{X_{0}}\right)\left(X_{p}-\overline{X_{p}}\right)}{\sqrt{\sum\left(X_{0}-\overline{X_{0}}\right)^{2}\left(X_{p}-\overline{X_{p}}\right)^{2}}}
\end{aligned}
$$

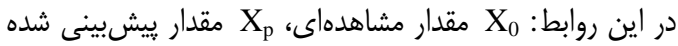

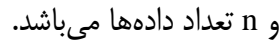

در اين تحقيق به منظور برآورد و ييشيينى تبخير-تعرق از دو حالت استفاده شد:

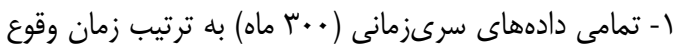

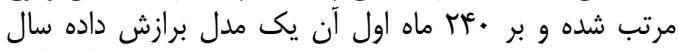

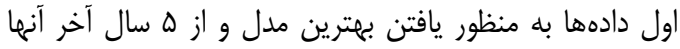
نيز جهت اعتبارسنجى نتايج مدل انتخابى استفاده كَرديد.

نتايج و بحث

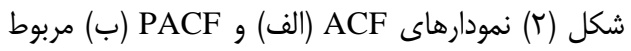

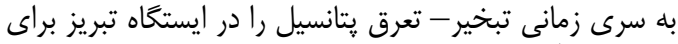

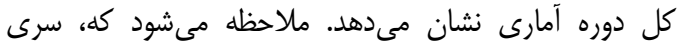

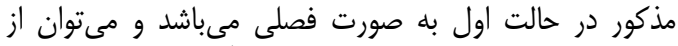

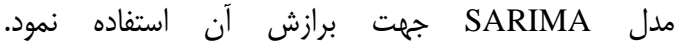

در اين رابطه q مرتبه مدل و م قها ضر ايب مدل مىباشند

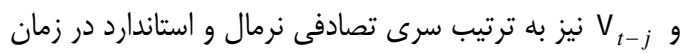
ا

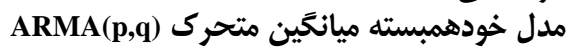

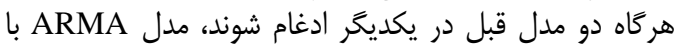

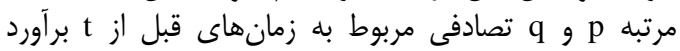

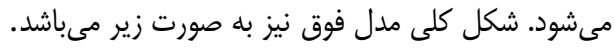
$Z_{t}=\sum_{i=1}^{P}\left(\varphi_{i} Z_{t-i}\right)-\sum_{i=1}^{q}\left(\theta_{j} \varepsilon_{t-j}\right)+\varepsilon_{t}$

كه در اين رابطه p مرتبه مدل AR و q مرتبه مدل MA و

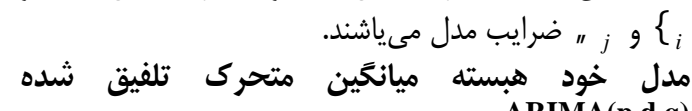
$\operatorname{ARIMA}(\mathbf{p}, \mathbf{d}, \mathbf{q})$

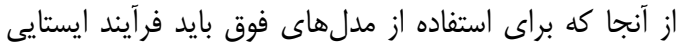

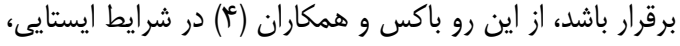

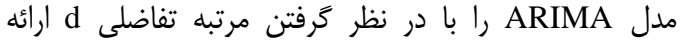

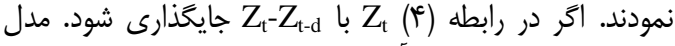
ARIMA بدست خواهد آمد. مدل خود

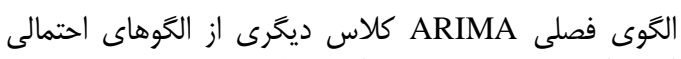

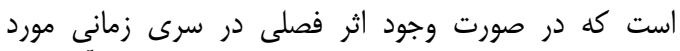

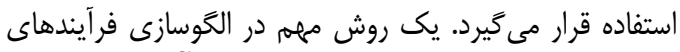

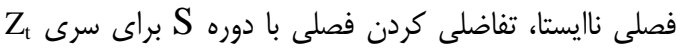

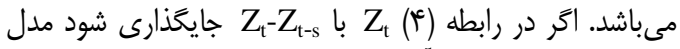
SARIMA

در اين تحقيق از مدلهاى SARIMA و ARIMA

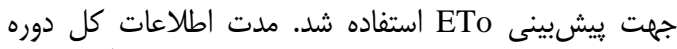

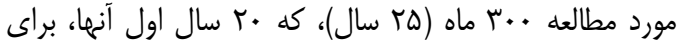
ييشبينى مدل و ها آخر سال نيز براى صحتسنجى مالى مدل

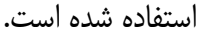



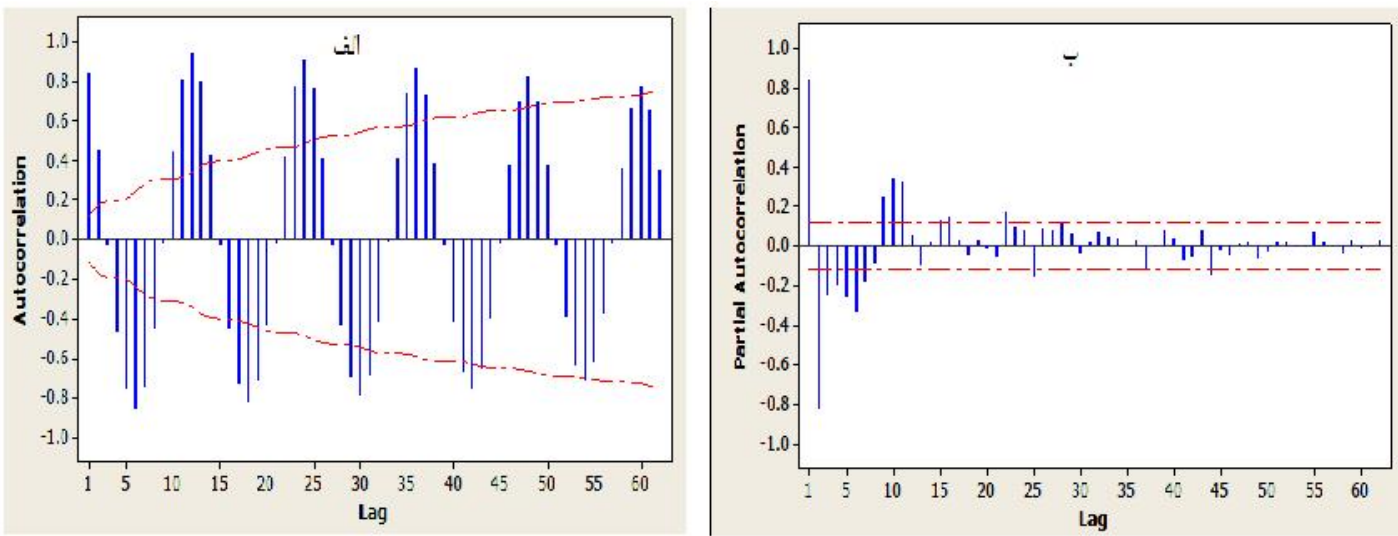

شكل r- نمودارهاى خودهمبستخى (الف) و خودهمبستخى جزئى (ب) سرىزمانى تبخير - تعرق در ايستخاه تبريز (كل دوره آمارى) Figure 2. Autocorrelation (a) and partial autocorrelation diagrams of evapotranspiration time series in Tabriz station. (The whole period statistical)

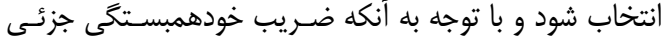
براى مرتبه اول از باند اطمينان خارج شده است، مرتبه

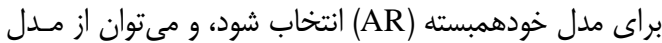

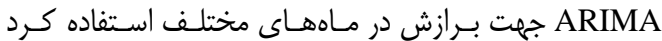

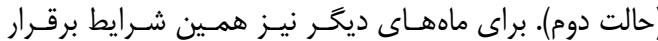

مىباشد.

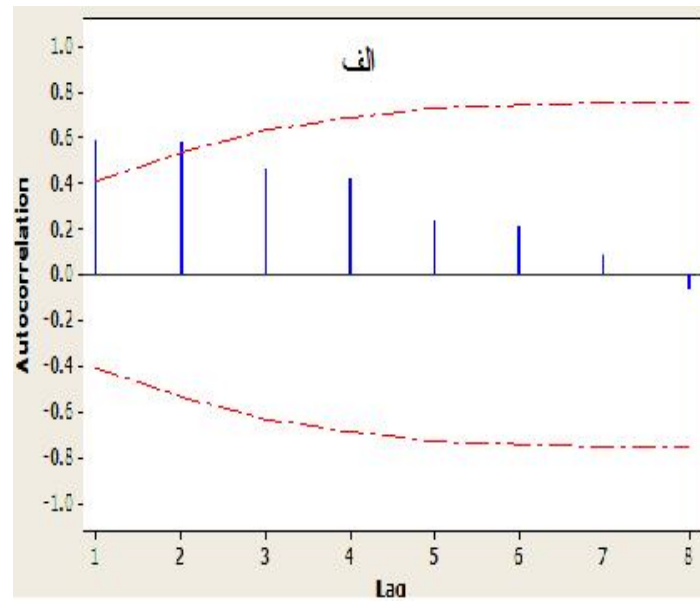

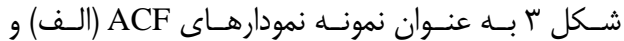

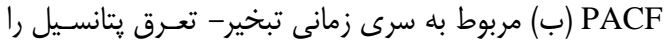

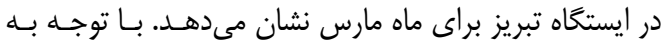

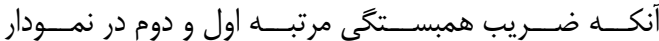

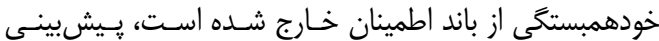

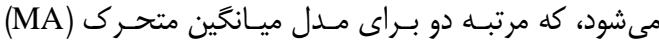

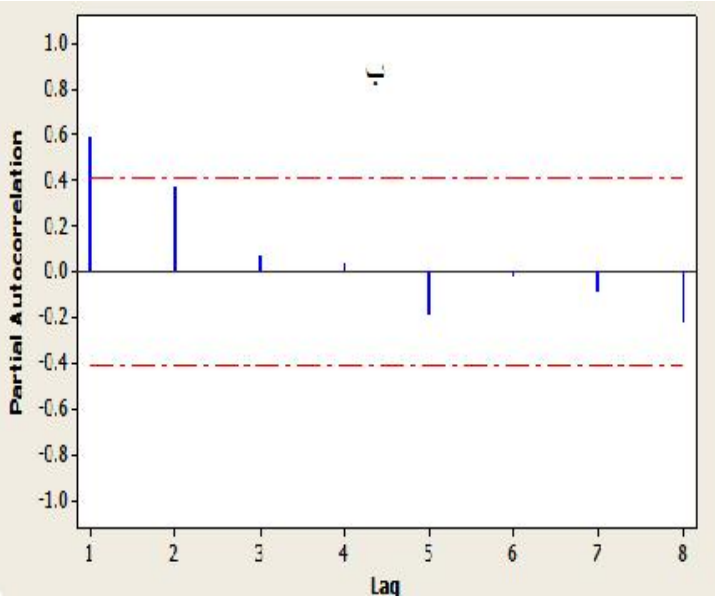

شكل ץ- نمودارهاى خودهمبستخى (الف) و خودهمبستخى جزئى (ب) سرىزمانى تبخير - تعرق در ايستخاه تبريز (ماه مارس)

Figure 3. . Autocorrelation (a) and partial autocorrelation diagrams of evapotranspiration time series in Tabriz station. (In march)

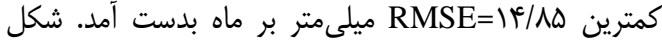

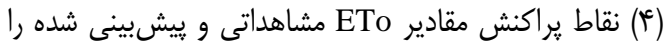

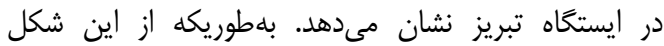

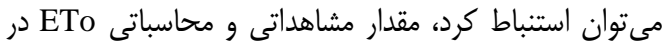

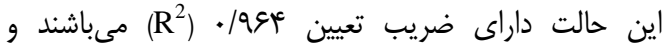

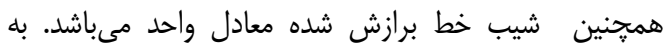

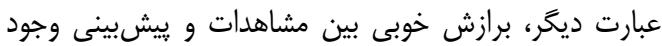

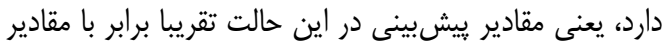

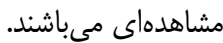

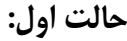

جهت مدلسازى و ييشبينى تبخير - تعرق در ايستخاه

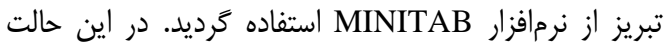

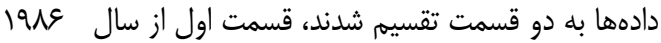

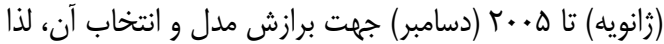

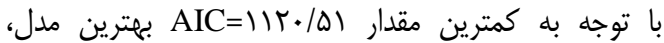
SARIMA(3,0,2).(0,1,4)

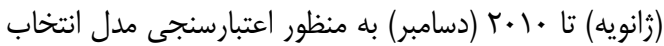

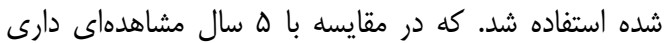




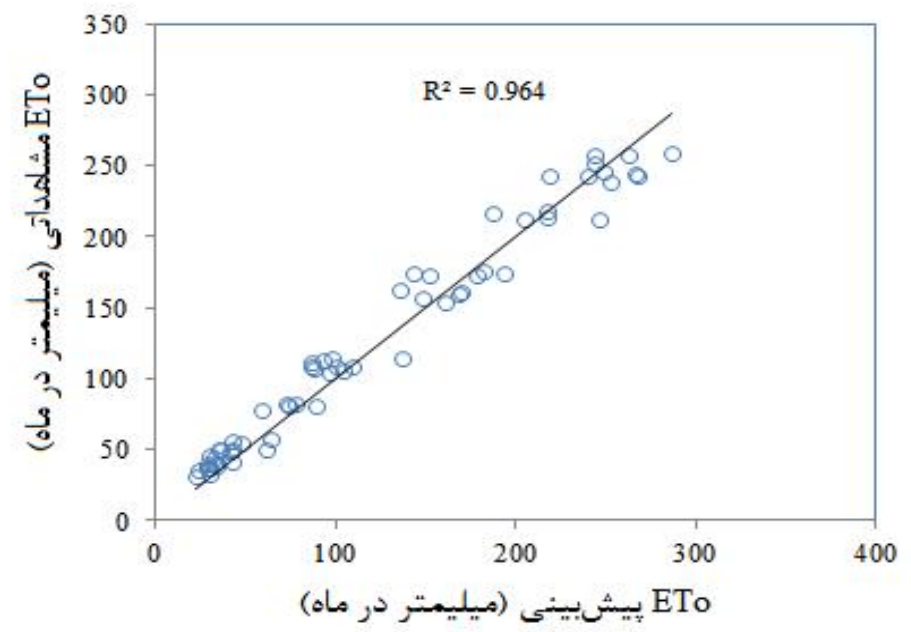

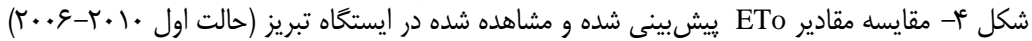

Figure 4. Comparison of the observed ETo and predicted ETo in Tabriz station. (The first approach, 2006-2010)

منى باشند. در نتيجه مدل برازش داده شده براى مدل صحيح

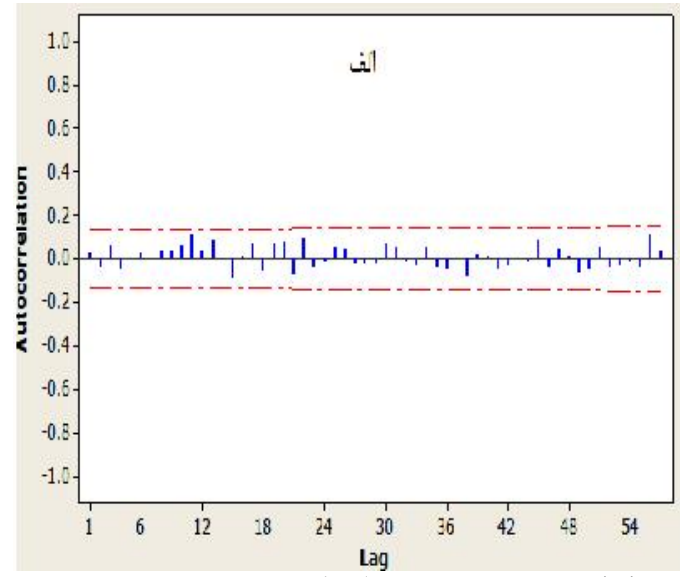

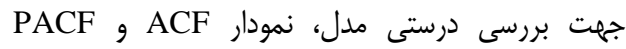
باقيماندها رسم كرديد (شكل هام). با توجه به ايه اين شكل در

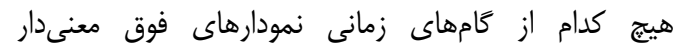

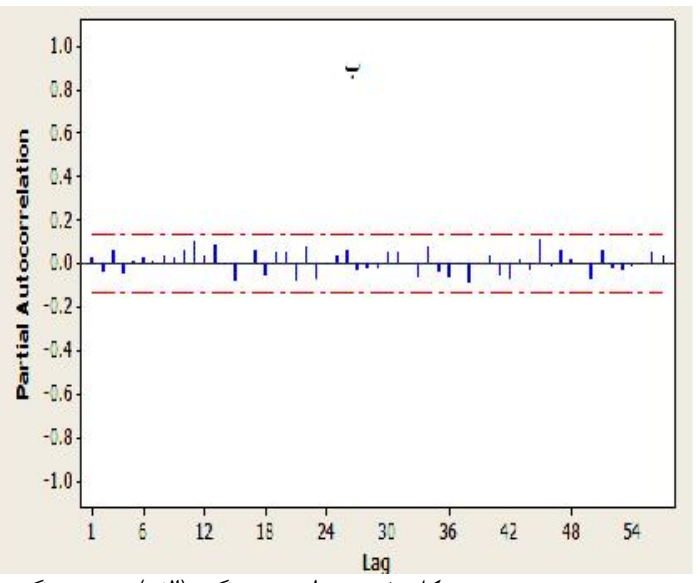

Figure 5. Autocorrelation (a) and partial autocorrelation (b) diagrams of ETo residues in Tabriz station

آمد. جدول (1) مشخصات بهترين مدل و همجنين مقادير

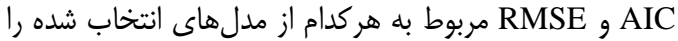

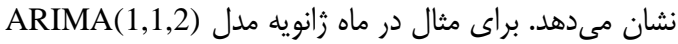

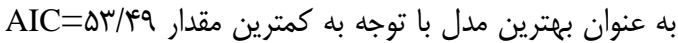

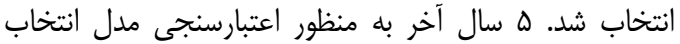

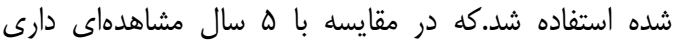

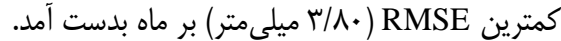

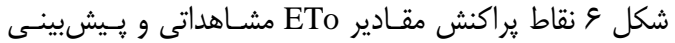

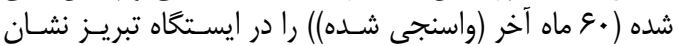

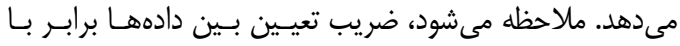

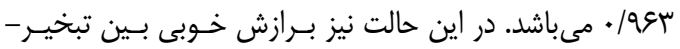

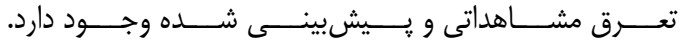

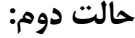

در اين حالت بيشبينى براى هر درام كدام از ماههاى سال

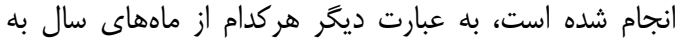

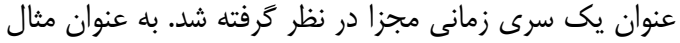

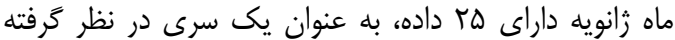

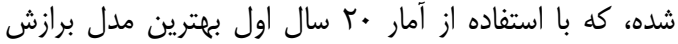

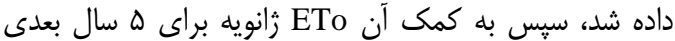

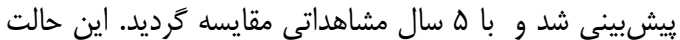

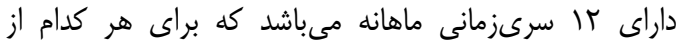

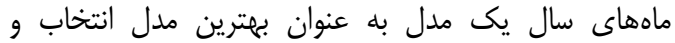

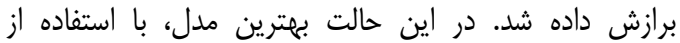

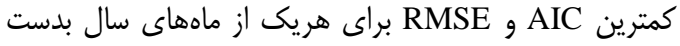




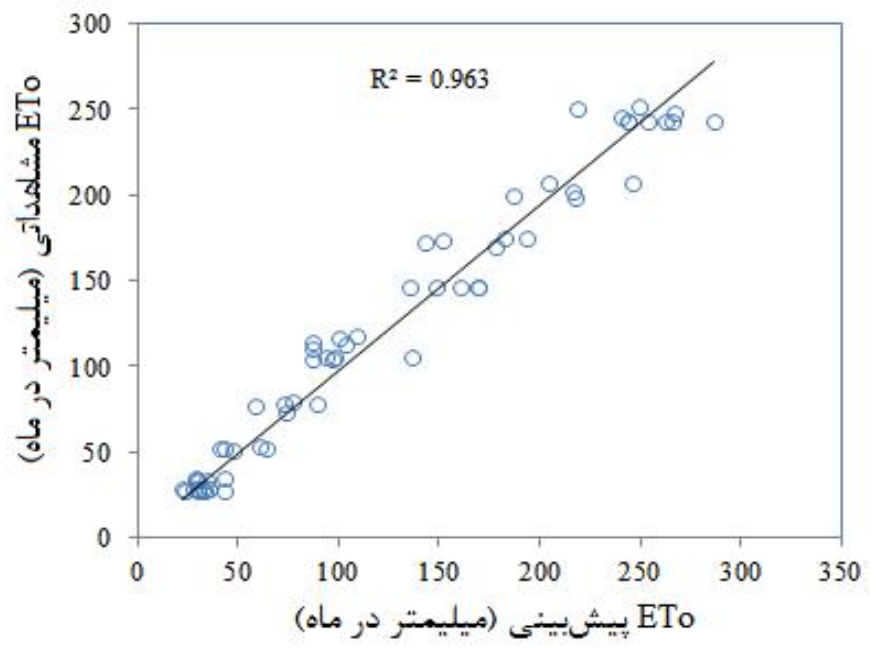

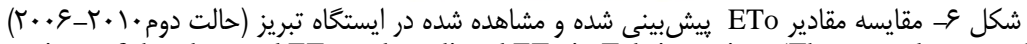

Figure 6. Comparison of the observed ETo and predicted ETo in Tabriz station. (The second approach, 2006-2010)

تاريخ وقوع مرتب گرديد و با دادههاى اصلى (ينج سال

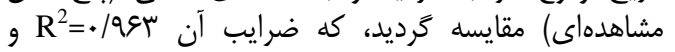

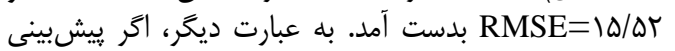

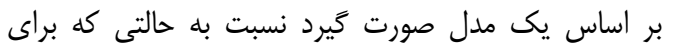

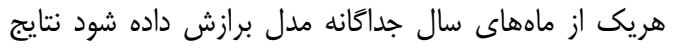

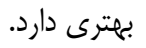

به منظور ارزيابى نتايج حاصل از دو روش مذكور، از معيارهاى ضريب تعيين و ريشه ميانخين مربعات خطا دارئ استفاده

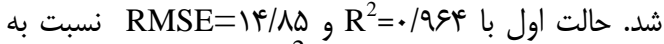

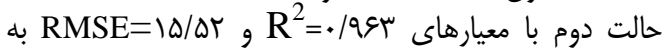

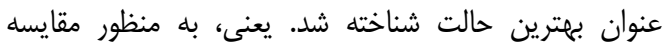

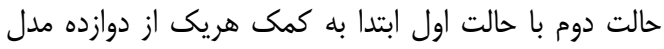

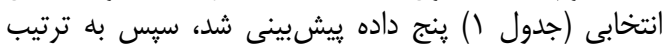

جدول ا- مقادير AIC و RMSE در مدلهاى مختلف سرىزمانى ARIMA

Table 1. AIC and RMSE mounts of ARIMA time series models

\begin{tabular}{|c|c|c|c|}
\hline RMSE (mm/month) & AIC & مدل & oto \\
\hline$r / \Lambda$. & $\Delta T / F q$ & $\operatorname{ARIMA}(1,1,2)$ & زرانويه \\
\hline N/ra & SD/IT & $\operatorname{ARIMA}(1,1,2)$ & فوريه \\
\hline $1 . / 1 T$ & NI/Gr & $\operatorname{ARIMA}(0,1,2)$ & مارس \\
\hline $\mid V / \& \Lambda$ & $৭ \vee / \Delta D$ & $\operatorname{ARIMA}(1,1,2)$ & آوريل \\
\hline$|8 / 9|$ & $1.9 / .9$ & $\operatorname{ARIMA}(1,0,0)$ & مى \\
\hline TI/אI & $119 / 90$ & $\operatorname{ARIMA}(1,0,2)$ & ز خوئن \\
\hline Tr/אTr & $\mid r \mu / r$ & $\operatorname{ARIMA}(1,0,0)$ & زويه \\
\hline $\mid V / r q$ & $111 / 49$ & $\operatorname{ARIMA}(2,1,2)$ & اوت \\
\hline 1N/9V & $\lambda r / \Delta)$ & $\operatorname{ARIMA}(2,1,2)$ & سيتامبر \\
\hline $\mid N / T V$ & $g F / M$ & $\operatorname{ARIMA}(1,1,2)$ & اكتبر \\
\hline $9 / 14$ & GY/M & $\operatorname{ARIMA}(0,1,3)$ & نوامبر \\
\hline$\Delta$ & $\Delta \cdot / \mu 1$ & $\operatorname{ARIMA}(0,1,3)$ & دسامبر \\
\hline
\end{tabular}

شكل V مقدار ETo مشاهداتى را با دو حالت مورد بررسى اول همريوشانى بهترى نسبت به مقادير ييشبينى در حالت دوم

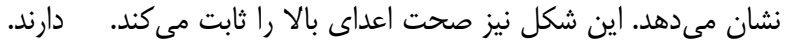

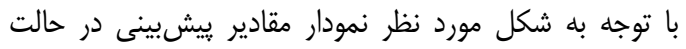




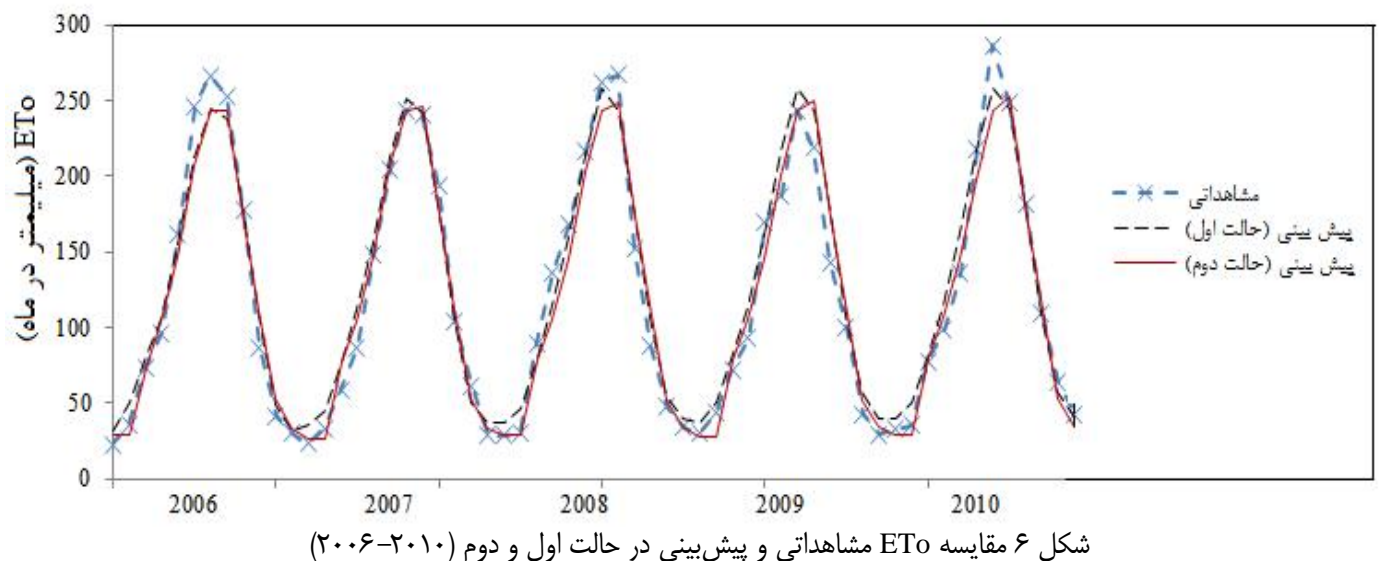

Figure 7. Comparison of the observed ETo and predicted ETo in the first and second approach

تبخير از تشت، مدل ARIMA(1,1,1) را ييشنهاد كردند، كـهـ

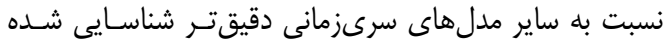

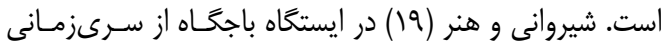
جهت SARIMA

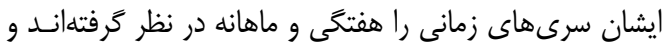

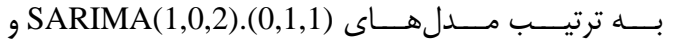

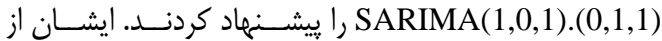

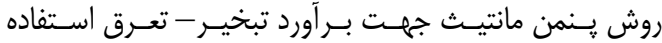

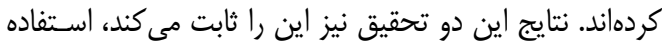

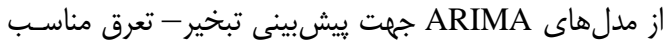

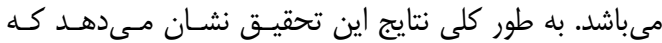

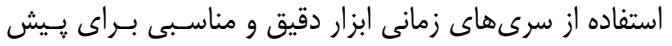
بينى مقادير تبخير- تعرق مىباشدا زماند
فرآيند تبخير- تعرق يكى از مولفههاى اصلى خرخه

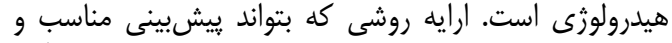

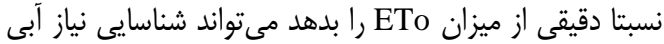

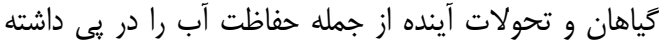

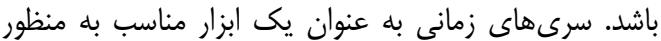

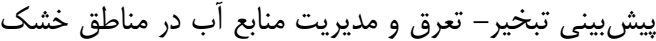

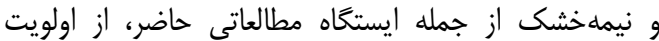
برخوردار هستند. توانمندى سرىزمانى ARIMA در تخمين

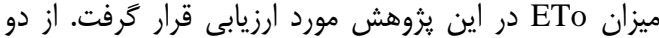

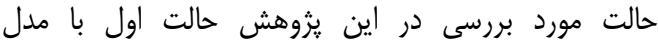

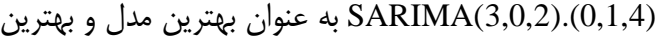

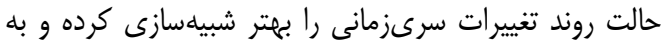

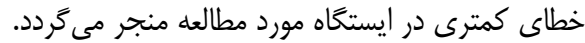

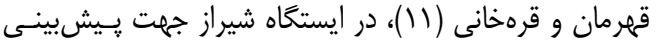




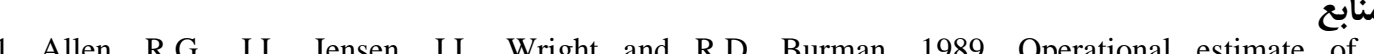
evapotranspiration. Agronomy Journal, 81: 650-662.

2. Allen, R.G., L.S. Pereira., D. Raes and M. Smith. 1998. Crop Evapotranspiration - Guidelines for Computing rop Water Requirements, FAO Irrigation and Drainage Paper 56, FAO, 1998, ISBN 92-5104219-5.

3. Babamiri, O., Y. Dinpashoh and E. Asadi. 2014. Calibration and evaluation of seven radiation- based reference crop evapotranspiration method at Urmia lake basin. Water and Soil Science, 23: 143-158 (In Persian).

4. Box, G. and G. Jenkins. 1976. Time series analysis forecasting and control. Holden-Day, San Francisco, 220-235.

5. Box, G., G. Jenkinks and G. Reinsel. 1994. Time series analysis: Forecasting and control. Third Edition, Holden-ay, 148-211.

6. Box, G. and D.R. Cox. 1964. An analysis of transformations, Journal of the Royal Statistical 26: 211 252.

7. Burlando, P., A. Montana and R. Raze. 1996. Forecasting of storm rainfall by combined use of radar, rain gages and linear models. Atmospheric Research, 42: 199-216.

8. Chattopahyay, N. and M. Hulme. 1997. Evaporation and potential evapotranspiration in India under conditions of recent and future climate change. Agricultural and Forest Meteorology, 87: 55-73.

9. Cohen S., A. Iantez and G. Stanhill. 2002. Evaporative climate changes at Bet Dagan 1964-1998. Agricultural and Forest Meteorology, 111: 83-91.

10. Dodangeh, A., J. Abedikoupai and A. Gohari. 2012. Application of Time Series Modeling to Investigate Future Climatic Parameters Trend for Water Resources Management Purposes. Journal of Water and Soil Science, Science and Technology of Agriculture and Natural Resources, 59: $59-17$ (In Persian).

11. Fooladmand, H. 2010.Monthly prediction of reference crop evapotranspiration in Fars province. Water and Soil Science, 1: 157-169 (In Persian).

12. Ghahreman, N. and A. Gharekhani. 2012. Evaluation stochastic time series models in pan evaporation estimating (case study Shiraz station). Journal of Water Research in Agriculture, 25. 1:75-81 (In Persian).

13. Gundekar, H.G., U.M. Khodke and S. Sarkar. 2008. Evaluation of pan coefficient for reference crop evapotranspiration for semi-arid region. Irrigation Science, 26: 169-175.

14. Hulme, M.Z., C. Zhao and T. Jiang. 1994. Recent and future climate change in East Asia. International Journal of Climatology, 14: 637-.658.

15. Kochekzade, M. and A. Bahmani. 2004. Evaluating the artificial neural network reducing the need parameters to archive reference evapotranspiration. Agricultural Sciences, 11:87-96.

16. Landeras, G., A. Ortiz-Barredo and J.J. López. 2009. Forecasting weekly evapotranspiration with ARIMA and artificial neural network models. Journal of Irrigation and Drainage Engineering, 135: 323-334.

17. Niroomand, H.A. 2009. Time series Analysis. Mashhad University, 145-220 (In Persian).

18. Peterson, T.C. 2002. Analysis of trends in US and the former USSR pan evaporation. National Climate Data Center. NOAA16:95-111.

19. Psilovikos, A. and M. Elhag. 2013. Forecasting of remotely sensed daily evapotranspiration data over Nile Delta Region, Egypt. Water Resources Management, 27: 4115-4130.

20. Salas, J.D. 1993. Analysis and modeling of hydrological time series. In: Handbook of Hydrology, Edited by Maidment, D.R, McGraw-Hill, New York, USA 410-455.

21. Shirwani, A. and T. Honar. 2012. Application of time series models for evapotranspiration forecasting in Bajgah station. Shahrekord University Electronic Journal Database, IWRJ. 8: 135-142 (In Persian).

22. Szilagy, J. 2001. Modeled area evaporation trend over the conterminous United States. Journal of Irrigation and Drainage Engineering, 127: 196-200.

23. Thomas, A. 2000. Spatial and temporal characteristics of potential evapotranspiration trends over China. International Journal of Climatology, 20: 381-396.

24. Zahedi, M., B. Sarisaraf and J. Jamei. 2006. Modeling the rainfall stations Urmia and Tabriz. Journal of Geography and Regional Development Research Journal, 7: 1-16 (In Persian). 


\title{
Potential Evapotranspiration Estimation using Stochastic Time Series Models (Case Study: Tabriz)
}

\author{
Omid Babamiri $^{1}$, Hamed Nowzari ${ }^{2}$ and Safar Maroofi ${ }^{3}$ \\ 1- PhD Student, Buali Sina University, (Corresponding Author: obabamiri@yahoo.com) \\ 2 and 3-Assistant Professor and Professor, Buali Sina University \\ Received: February 13, $2016 \quad$ Accepted: June 20, 2016
}

\begin{abstract}
Evapotranspiration is important components of hydrological cycle, which is important in irrigation systems planning and evaluation of climate change impacts on water planning. In this study, evapotranspiration time series using Penman Monteith was studied in Tabriz synoptic station by the linear stochastic models such as ARIMA and SARIMA. The data had been used since 1986 to 2010. After calculating evapotranspiration, the first 20 years and last 5 years were used for model calibration and validation, respectively. This research was performed in two distinguish approaches. In the first approach all data were considered as unit series with an appropriate fitting model. In the second approach, the monthly series of using data were selected, separately. Then, for each month an appropriate model were fitted to data. The results showed that, the first approach was recognized as a better method regarded the second approach. The $\mathrm{R}^{2}$ and RMSE values were 0.964 and 14.85 first approach comparing to them of 0.963 and 15.52 in the second approach. In conclusion, the $\mathrm{R}^{2}$ and RMSE values of the approaches were relatively similar, with very small $(0.67 \mathrm{~mm} / \mathrm{month})$ better error rate. In whole the two approaches don't have significant difference but the first approach is recommendable because it includes fewer computations.
\end{abstract}

Keywords: ARIMA, Forecast, Penman Monteith and SARIMA 